\title{
The phase separation effects in a pseudospin-electron model
}

\author{
O.D.Danyliv, I.V.Stasyuk \\ Institute for Condensed Matter Physics \\ of the National Academy of Sciences of Ukraine, \\ 1 Svientsitskii Str., 79011 Lviv, Ukraine
}

Received December 25, 2001

The two-sublattice pseudospin-electron model of high temperature superconductors is studied with respect to phase separations. Such a model can be used for the description of dielectric properties of YBaCuO-type crystals along $c$-axis (the pseudospins represent an anharmonic motions of apical oxygen O4). The model is treated within different approximations. It is shown that the model has got phase transitions to the phase with pseudospin ordering as well as phase separation. The effect of separation on the appearance of the ordered state is discussed.

Key words: phase separation, pseudospin, ferroelectric ordering

PACS: $74.65 .+n, 71.45 . \mathrm{Gm}$

\section{Introduction}

The role of apex oxygen $\mathrm{O} 4$ in high $\mathrm{T}_{\mathrm{c}}$ superconductors is not well studied yet as well as the mechanism of high values of superconducting phase transition temperatures is not quite clear. The analysis of EXAFS and Raman spectra of the most studied superconductor $\mathrm{YBa}_{2} \mathrm{Cu}_{3} \mathrm{O}_{7-\delta}[1-3]$ reveals the existence of a strongly anharmonic double well potential of apex oxygen. This conclusion agrees with the results of X-rays diffraction experiments [4]. In some experiments $\mathrm{YBaCuO}$ was found to be both pyroelectric and piezoelectric, implying the existence of macroscopic polarization directed along the $c$-axis [5]. This feature was connected with the existence of anharmonic vibrations of O4. However, in [6] an alternative interpretation of EXAFS data was given: it was suggested that the two-site $\mathrm{O} 4$ configurations may be related to strong local distortions around single chain O1-vacancies.

To take into account the strong on-site Coulomb interaction in $\mathrm{CuO}$ planes and the interaction of electrons with local vibrations of apex oxygen the pseudospinelectron model (PEM) was used [7]. To explain the possible phase transitions con- 
nected with the apex oxygen, it was proposed to consider the long range pseudospinpseudospin interaction [8]. To correctly describe the dielectric anomalies, PEM was extended to a two-sublattice case [10].

The purpose of the current paper is to consider PEM from the point of view of the phase separation and to study the influence of a separated state on the ferroelectric type ordering which can be present it the system.

\section{The Hamiltonian of model}

In the paper we investigate the dielectric and thermodynamic properties of the two-sublattice PEM, which takes into account a two-sublattice structure of an elementary cell of $\mathrm{YBaCuO}$-type superconductors. Within the framework of this model, the Hubbard model (applied to the description of electrons) is supplemented by a pseudospin subsystem $[9,10]$ :

$$
\begin{aligned}
H & =H_{\mathrm{e}}+H_{\mathrm{s}}+H_{\mathrm{e}-\mathrm{s}}+H_{\mathrm{s}-\mathrm{s}} \\
H_{\mathrm{e}} & =-\mu \sum_{n, s}\left(n_{n 1}^{s}+n_{n 2}^{s}\right)+U \sum_{n}\left(n_{n 1}^{\uparrow} n_{n 1}^{\downarrow}+n_{n 2}^{\uparrow} n_{n 2}^{\downarrow}\right), \\
H_{\mathrm{s}} & =-h \sum_{n}\left(S_{n 1}^{z}-S_{n 2}^{z}\right), \\
H_{\mathrm{e}-\mathrm{s}} & =g \sum_{n, s}\left(n_{n 1}^{s} S_{n 1}^{z}-n_{n 2}^{s} S_{n 2}^{z}\right), \\
H_{\mathrm{s}-\mathrm{s}} & =-J \sum_{n} S_{n 1}^{z} S_{n 2}^{z}-\frac{1}{2} \sum_{n, n^{\prime}} \sum_{\alpha, \beta} J_{n n^{\prime}}^{\alpha \beta} S_{n \alpha}^{z} S_{n^{\prime} \beta}^{z} .
\end{aligned}
$$

Here, $n_{n \alpha}^{s}$ is the operator of the number of electrons with a spin $s$ while $S_{n \alpha}^{z}$ stands for the operator of the pseudospin at the $n$ cell in $\alpha$ plane $(\alpha=1,2$ in the two-sublattice case). $H_{\mathrm{e}}$ is the Hubbard Hamiltonian without a term describing the transfer of electrons. $H_{\mathrm{s}}$ is the pseudospin part of the Hamiltonian and $h$ describes the asymmetry of the well potential. $H_{\mathrm{e}-\mathrm{s}}$ is the term describing the interaction between electrons and pseudospins. $H_{\mathrm{s}-\mathrm{s}}$ gives the interaction between pseudospins; the interaction $-J S_{n 1}^{z} S_{n 2}^{z}$ within one cell clusters is separated.

It is convenient to study the Hamiltonian based on the states $|i, K\rangle \equiv|i, 1, R\rangle \otimes$ $|i, 2, S\rangle$ which is the product of one-site states $|i, \alpha, R\rangle \equiv\left|\hat{n}_{i \alpha}^{\uparrow}, \hat{n}_{i \alpha}^{\downarrow}, \hat{S}_{i \alpha}^{z}\right\rangle \quad(\alpha=1,2)$. Each one-site set of states includes eight components at a given site $i$ and sublattice $\alpha:$

$$
\begin{array}{rlrl}
|1\rangle & =|0,0, \uparrow\rangle, & & |\tilde{1}\rangle=|0,0, \downarrow\rangle, \\
|2\rangle=|1,1, \uparrow\rangle, & & |\tilde{2}\rangle=|1,1, \downarrow\rangle, \\
|3\rangle & =|0,1, \uparrow\rangle, & & |\tilde{3}\rangle=|0,1, \downarrow\rangle, \\
|4\rangle & =|1,0, \uparrow\rangle, & & |\tilde{4}\rangle=|1,0, \downarrow\rangle .
\end{array}
$$

Hereafter we will consider the case of infinitely large Coulomb repulsion $U$. It means that we are able not to consider the doubly occupied states $|2\rangle$ and $|\tilde{2}\rangle$. 

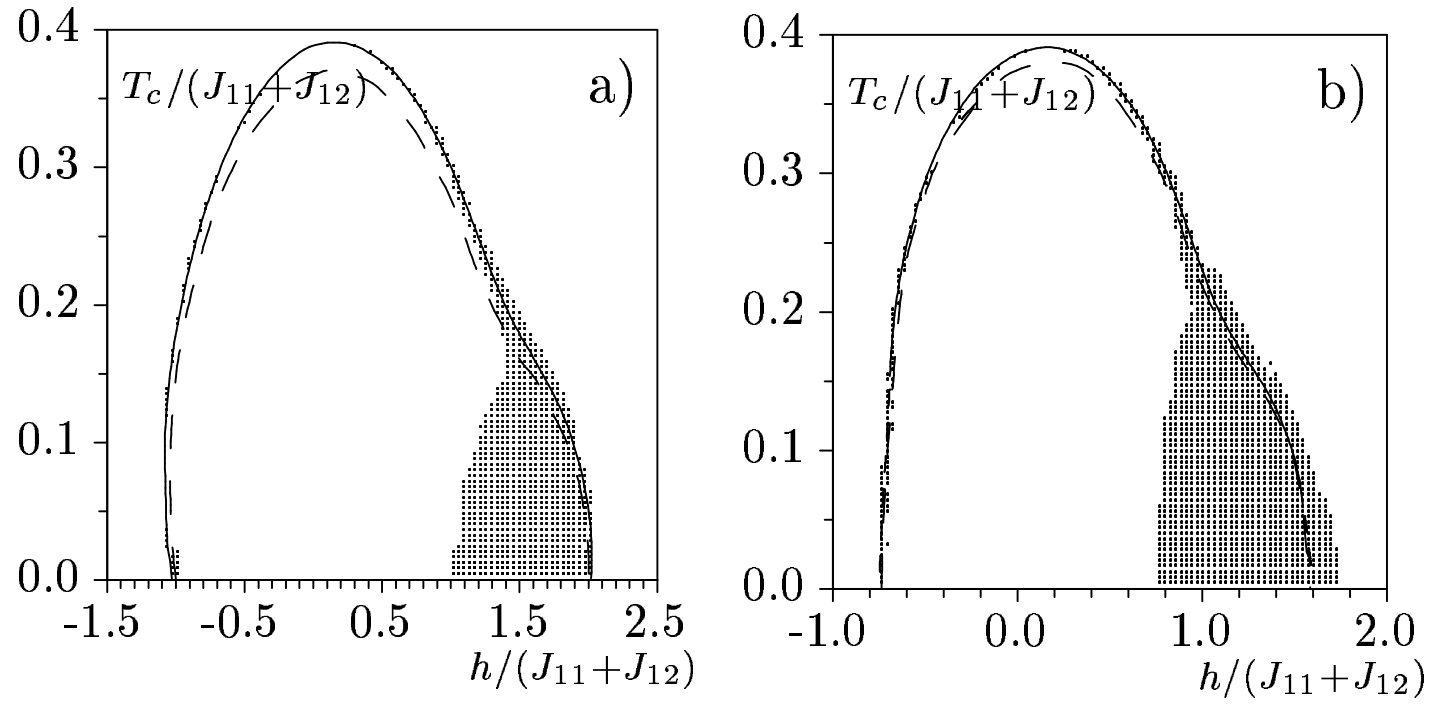

Figure 1. $h$ dependence of the temperature of ferroelectric phase transition $T_{\mathrm{c}}$ at different values of parameter $J_{11}-J_{12}$ in the regime $n=$ const: a) $\left(J_{11}-J_{12}\right) /\left(J_{11}+\right.$ $\left.J_{12}\right)=-1$, b) $\left(J_{11}-J_{12}\right) /\left(J_{11}+J_{12}\right)=0$. Other parameters: $J /\left(J_{11}+J_{12}\right)=1$, $g /\left(J_{11}+J_{12}\right)=1, n=0.4$. Solid lines and dashed lines represent the second order and the first order phase transitions, respectively. The widely spaced dashed line corresponds to a one-loop approximation. Dots represent a separation area.

\section{Phase separation induced by pseudospin-pseudospin interaction}

In [10] the phase transitions into ferroelectric phase (with the order parameter $\eta=1 / N \sum_{n}\left\langle S_{n 1}^{z}-S_{n 2}^{z}\right\rangle$ describing a macroscopic polarization) were investigated in the mean field approximation and in the one-loop approximation. The regions of parameter values of model (1) at which the ferrolelectric ordering takes place were found. Nevertheless, the phase separation processes were not taken into account, as far as they were found at zero temperature in the simple (one-sublattice) pseudospinelectron model [12].

Figure 1 presents the phase diagrams for model (1) at different values of long range interaction $J_{\alpha \beta}=\sum_{n^{\prime}} J_{n n^{\prime}}^{\alpha \beta}$ and fixed concentration $n=0.4$. In areas marked by points, the system is separated into two regions with concentrations $n_{1}$ and $n_{2}$ $\left(n_{1}<n<n_{2}\right)$. Also, one can notice that phase separation takes place near the border of stability region of two phases (the ordered with nonzero polarization and the disordered). That is why the ordered phase becomes wider and is extended up to the edge of the separated area. Figure 2 illustrates such a behaviour. The dashed lines here represent the region of ferroelectric type instabilities. These lines would separate the ferroelectric phase if there was no phase separation. Figure $2 \mathrm{~b}$ also shows that at a fixed value of anharmonicity parameter $h$ with concentration $n<0.75$ (at low temperature), the ordered phase is possible only because the phase separation takes place. 

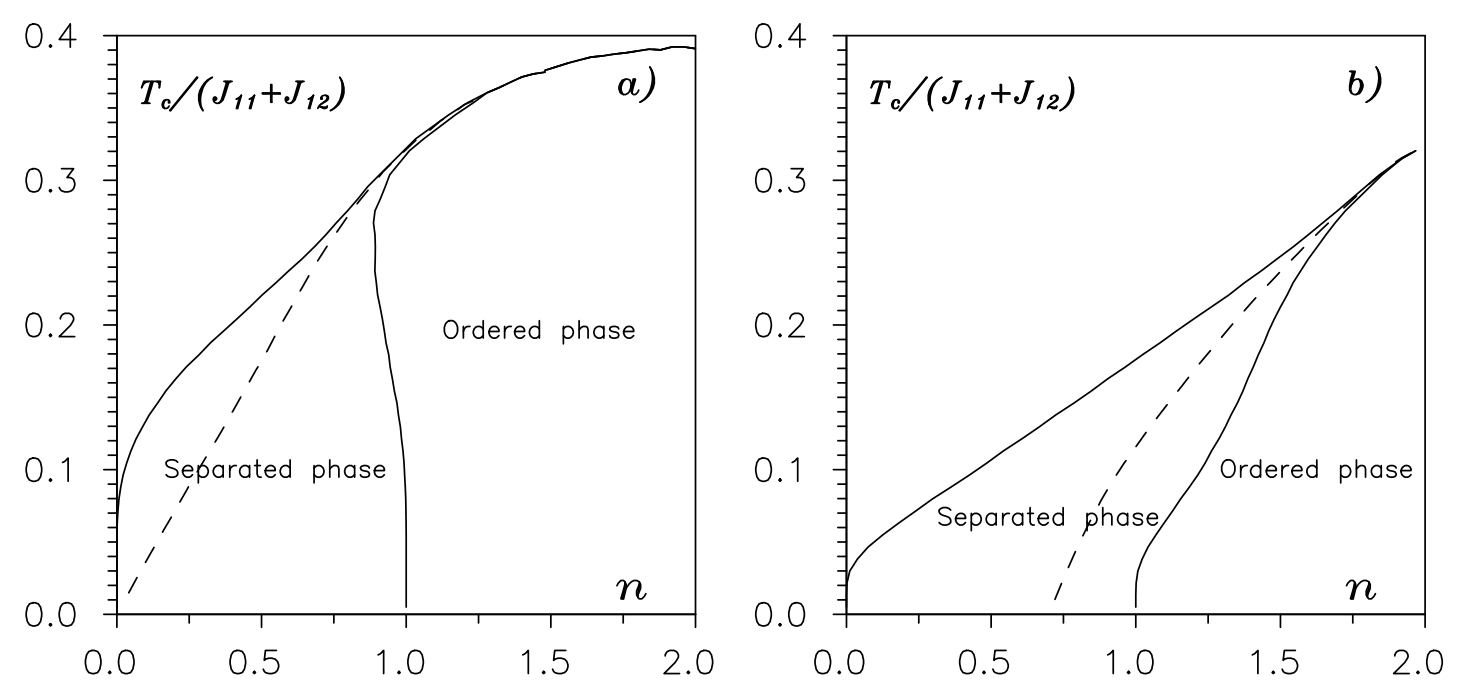

Figure 2. Phase $T-n$ diagram in a mean field approximation. The phase separation region is limited by solid lines. The dashed lines point to the region of ferroelectric instabilities. Parameters: $\left(J_{11}-J_{12}\right) /\left(J_{11}+J_{12}\right)=1, J /\left(J_{11}+J_{12}\right)=$ $g /\left(J_{11}+J_{12}\right)=1 ;$ a $) h /\left(J_{11}+J_{12}\right)=1$, b) $h /\left(J_{11}+J_{12}\right)=1.35$.

\section{The role of electron transfer}

Let us now consider the effect of electron transfer on the phase transitions. It has been shown (in the simplest approximation Hubbard-I [11]) that at $U \rightarrow \infty$ in a one-sublattice case of model (1), one has in Hubbard-I approximation two subbands, separated by the gap equal to $g$ :

$$
\begin{aligned}
& \varepsilon_{\alpha}^{31}(\mathbf{k})=\varepsilon_{\alpha}^{41}(\mathbf{k})=-(-1)^{\alpha} \frac{g}{2}+t_{\mathbf{k}}\left\langle X_{\alpha}^{44}+X_{\alpha}^{11}\right\rangle_{0}, \\
& \varepsilon_{\alpha}^{\tilde{3} \tilde{1}}(\mathbf{k})=\varepsilon_{\alpha}^{\tilde{4} \tilde{1}}(\mathbf{k})=(-1)^{\alpha} \frac{g}{2}+t_{\mathbf{k}}\left\langle X_{\alpha}^{\tilde{4} \tilde{4}}+X_{\alpha}^{\tilde{1} \tilde{1}}\right\rangle_{0} .
\end{aligned}
$$

Here, $\langle\ldots\rangle_{0}$ represents an average over one-site part of Hamiltonian (1), $t_{\mathbf{k}}=$ $\frac{1}{N} \sum_{i-j} t_{i j} \mathrm{e}^{\mathrm{ikr} \mathbf{r}_{i j}}-$ Fourier transformation of hopping integral and $X_{i \alpha}^{R S}=|i, \alpha, R\rangle\langle i, \alpha, S|$ are Hubbard operators on the basis of states (2). We can improve this result following the self-consistent generalized random phase approximation [13], which corresponds to the summing of all one-loop contributions to $X^{p p}$ in diagrammatic representation. It gives us a shift of subbands by $\tilde{Q}^{p q}$; the mean values of the Hubbard operators then should be found self-consistently.

The changes in excitation spectrum (3) are demonstrated in figure 3b. There is no more symmetry $\varepsilon_{1}^{p q}(\mathbf{k})=-\varepsilon_{2}^{p q}(\mathbf{k})$. It is due to ferroelectric type ordering in the system. The chemical potential behaviour near the edge of the band points to the appearance of a phase separation. Figure 3 shows that in the case when the solution of the system of equations indicates that the ferroelectric phase appears (figure 3a), the separation takes place before that. This is also indicated by a certain concavity in the free energy behaviour (tangent dashed lines in figure $3 \mathrm{c}$ link the points with 

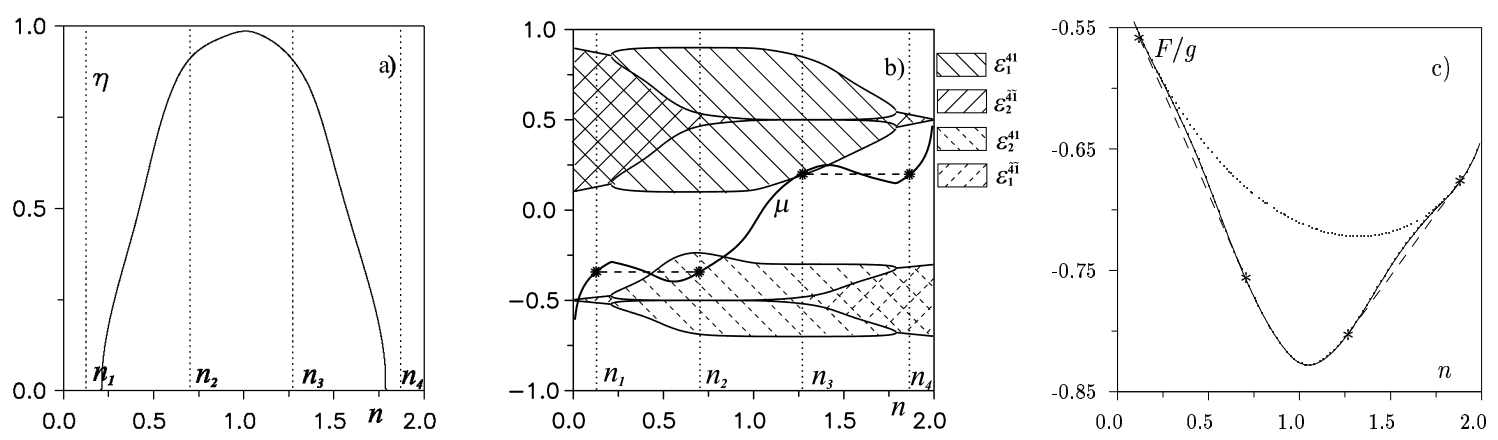

Figure 3. Dependence of the order parameter $\eta$ (a), band spectrum (b), and free energy (c) on concentration. The parameter values are: $J_{11}=J_{12}=g / 2$, $T / g=0.1, t_{i j} / g=0.1, h / g=0.5$.
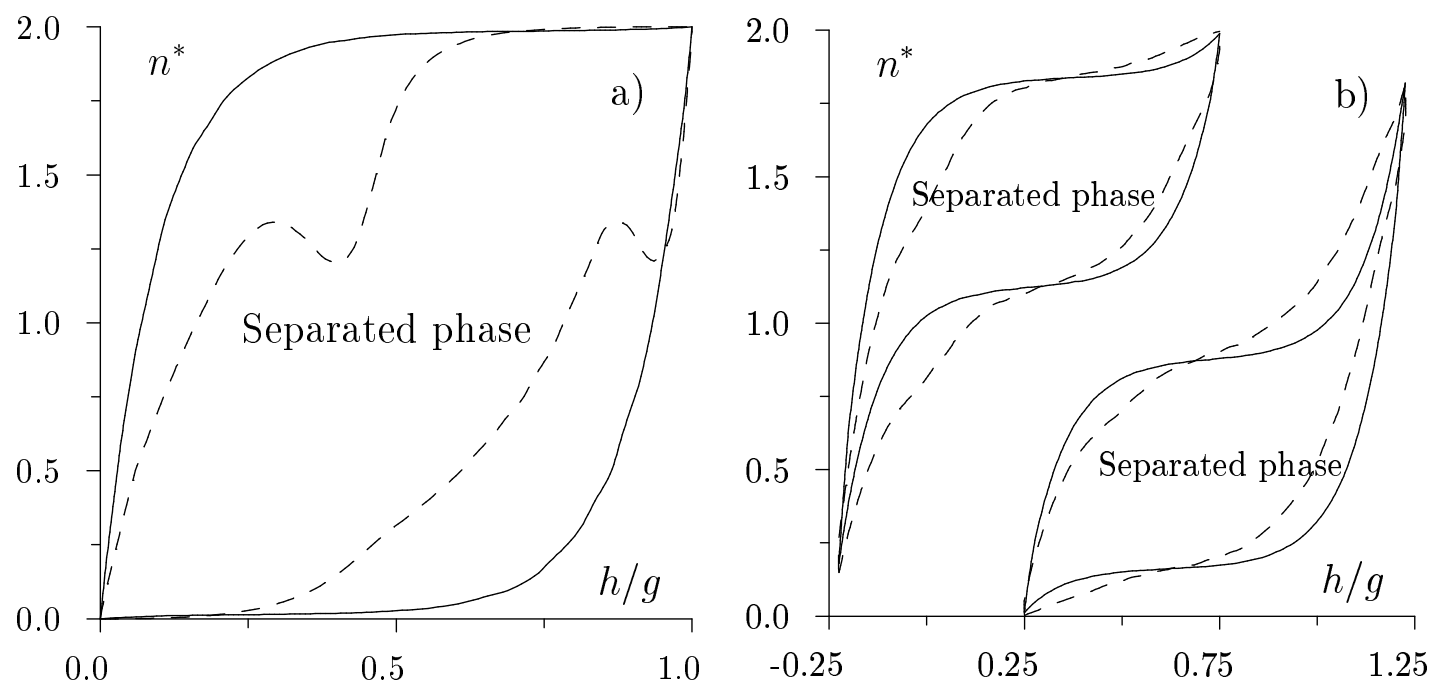

Figure 4. Regions of phase separation at different values of interaction $J_{\alpha \beta}, T / g=$ 0.1 . Thick lines correspond to the case $t_{i j}=0$; dashed lines correspond the the case $t_{i j} \neq 0$. a) $J_{11}=g, J_{12}=0, t_{i j} / g=0.2$; b) $J_{11}=J_{12}=g / 2, t_{i j} / g=0.1$.

concentration values $n_{1}, n_{2}$ and $n_{3}, n_{4}$ on which the separation takes place). Hence, there is a separation in the paraelectric and ferroelectric phases at concentration values $n_{1}<n<n_{2}$ and $n_{3}<n<n_{4}$. The pure ferroelectric phase remains in the concentration range $n_{2}<n<n_{3}$.

Figure $4 \mathrm{a}$ and $4 \mathrm{~b}$ show the boundaries of the separated phase in the presence and in the absence of electron transfer. Their positions are temperature dependent. At $T=0$ the region of phase separation reaches the sides of the $0 \leqslant h / g \leqslant 1$, $0 \leqslant n^{*} \leqslant 2[12]$ rectangle. The transfer effect is ambiguous. In the first case (4a) it narrows the region of separation, whereas in the second case (4b) the transfer only changes the shape of the region boundaries. 


\section{Conclusions}

It is shown that the phase transition to the ordered polar phase in the twosublattice pseudospin-electron model can be accompanied by a phase separation. The system is separated into the regions with a different value of concentration and polarization. The phase separation widens the area of the model parameter values where polarization takes place: the borders of a separated phase become new borders of the region with nonzero order parameter $\eta$.

It is shown that electron transfer has an effect on the phase separation caused by pseudospin-pseudospin interaction. Electron hopping changes the shape of the region boundaries. The reverse effect also takes place: the electron spectrum is changed by a phase separation.

The obtained results correlate well with the experimental data on neutron scattering for high- $T_{\mathrm{c}}$ superconductors which indicate the existence of stripe phases in $\mathrm{La}_{2-x} \mathrm{Sr}_{x} \mathrm{CuO}_{4}[14]$ as well as in $\mathrm{YBa}_{2} \mathrm{Cu}_{3} \mathrm{O}_{7-\delta}$ [15]. Also, there is a problem of a genesis of structural inhomogeneities in a single crystal of the $\mathrm{YBaCuO}$ type observed by the experiments using Raman spectroscopy [16] and mesoscopic structural date [17]. Our results show the possibility of dielectric type mechanism of the appearance of the mentioned instabilities.

\section{Acknowledgement}

This work was partially supported by the Fundamental Research Fund of the Ministry of Ukraine for Science and Education (Project No. 02.07/266).

\section{References}

1. Maruyama H., Ishii T., Bamba N., Maeda H., Koizumi A., Yoshikawa Y., Yamazaki H. // Physica C, 1989, vol. 60, No. 5/6, p. 524.

2. Mustre de Leon J., Conradson S.D., Batistic' I., Bishop A.R., Raistrick I.D., Aronson M.C., Garzon F.H. // Phys. Rev. B, 1992, vol. 45, p. 2447.

3. Ruani G., Taliani C., Muccini M., Conder K., Kaldis E., Keller H., Zech D., Muller K.A. // Physica C, 1994, vol. 226, p. 101.

4. Conradson S.D., Raistrick I.D. // Science, 1989, vol. 243, No. 4896, p. 1340.

5. Mihailovic D., Heeger A.J. // Solid State Comm., 1990, vol. 75, p. 319.

6. Röhler J. - In: Kaldis E. (ed.), Materials and crystallographic aspects of $\mathrm{HT}_{\mathrm{c}^{-}}$ superconductivity. The Netherlands, Kluwer Academic Publishers, 1994, p. 353.

7. Stasyuk I.V., Shvaika A.M., Schachinger E. // Physica C, 1993, vol. 213, No. 1, p. 57.

8. Galbaatar T., Plakida N.M. // Proc. Intern. Conference $\mathrm{M}^{2} \mathrm{~S}-\mathrm{HTSC}$ IV, 5-9 July, 1994, Grenoble.

9. Danyliv O.D., Stasyuk I.V. // Condens. Matter Phys., 1996, No. 7, p. 163.

10. Danyliv O.D. // Physica C, 1998, vol. 309, p. 303.

11. Stasyuk I.V., Shvaika A.M. // Acta Physica Polonica A, 1993, vol. 84, No. 2, p. 293.

12. Stasyuk I.V., Havrylyuk Yu. // Condens. Matter Phys., 1999, vol. 2, No. 3(19), p. 487494. 
13. Stasyuk I.V., Danyliv O.D. // Phys. stat. sol. (b), 2000, vol. 219, p. 299.

14. Emery V.J., Kivelson, S.A., Tranquada J.M. Preprint cond-matt/9907228, 1999.

15. Guttmann M., Billinge S.J.L., Brosha E.L., Kwei G.H. Preprint cond-matt/9908365, 1999.

16. Iliev M.N., Hadjiev V.G., Ivanov V.G. // Journ. Raman. Spectr., 1996, vol. 27, p. 333.

17. Browning V.M. // Phys. Rev. B, 1997, vol. 65, p. 2860.

\section{Ефекти фазового розшарування у псевдоспін-електронній моделі}

\section{О.Д.Данилів, І.В.Стасюк}

Інститут фізики конденсованих систем НАН України, 79011 Львів, вул. Свєнціцького, 1

\section{Отримано 25 грудня 2001 р.}

Розглянуто двопідґраткову псевдоспін-електронну модель високотемпературних надпровідників з огляду на можливість появи фазових розшарувань. Така модель здатна описати діелектричні властивості кристалів типу ҮВаСuО вздовж осі $c$ (псевдоспінами представлено ангармонічні локальні коливання вершинного кисню О4). Модель досліджено в різних наближеннях. Показано, що поряд з фазовим переходом у фазу із впорядкованою псевдоспіновою системою в моделі присутнє фазове розшарування. Обговорюється вплив розшарування на появу впорядкованої фази.

Ключові слова: фазове розшарування, псевдоспін, сегнетоелектричне впорядкування

PACS: $74.65 .+n, 71.45 . \mathrm{Gm}$ 
